\title{
Indoleamine 2, 3-dioxygenase (IDO) is essential for dendritic cell activation and chemotactic responsiveness to chemokines
}

\author{
Shih Ling HWANG ${ }^{1}$, Nancy Pei-Yee CHUNG ${ }^{2}$, Jacqueline Kwai-Yi CHAN², Chen-Lung Steve LIN 2,* \\ ${ }^{1}$ Department of Surgery, Kaohsiung Medical University, Kaohsiung, Taiwan, China \\ ${ }^{2}$ Department of Surgery, The University of Hong Kong Medical Centre, Queen Mary Hospital, Pokfulam Road, Hong Kong \\ SAR, China
}

\begin{abstract}
Indoleamine 2, 3-dioxygenase (IDO) is a rate-limiting enzyme for the tryptophan catabolism. In human and murine cells, IDO inhibits antigen-specific T cell proliferation in vitro and suppresses $\mathrm{T}$ cell responses to fetal alloantigens during murine pregnancy. In mice, IDO expression is an inducible feature of specific subsets of dendritic cells (DCs), and is important for T cell regulatory properties. However, the effect of IDO and tryptophan deprivation on DC functions remains unknown. We report here that when tryptophan utilization was prevented by a pharmacological inhibitor of IDO, 1-methyl tryptophan (1MT), DC activation induced by pathogenic stimulus lipopolysaccharide (LPS) or inflammatory cytokine TNF- $\alpha$ was inhibited both phenotypically and functionally. Such an effect was less remarkable when DC was stimulated by a physiological stimulus, CD40 ligand. Tryptophan deprivation during DC activation also regulated the expression of CCR5 and CXCR4, as well as DC responsiveness to chemokines. These results suggest that tryptophan usage in the microenvironment is essential for DC maturation, and may also play a role in the regulation of DC migratory behaviors.
\end{abstract}

Keywards: Indoleamine 2,3-dioxygenase (IDO), dendritic cells, activation, T cell, tryptophan, chemokine.

\section{INTRODUCTION}

Indoleamine 2,3-dioxygenase (IDO) is a rate-limiting enzyme that degrades the essential amino acid tryptophan into kynurenine [1]. It has also been postulated that the role of IDO is to inhibit the proliferation of eukaryotic intracellular pathogens $[2,3]$ or tumour cells [4] by depriving them of tryptophan. In these settings, the proposed role of IDO has been to eliminate the cell's own stores of tryptophan. Although poorly understood, there are also intriguing associations between altered tryptophan metabolism and immunological phenomena. For example, patients receiving 5-hydroxy-tryptophan for neurological disorders experienced an unexpected high frequency of a

*Correspondence: Chen-Lung Steve LIN

E-mail: clin@hkucc.hku.hk

Abbreviations: IDO, Indoleamine 2,3-dioxygenase; 1MT, 1-methyl tryptophan; DCs, dendritic cells; CCR, CC chemokine receptor; CXCR, CXC chemokine receptor; RANTES, regulated upon activation, normal T-cell expressed, and presumably secreted; MIP, macrophage inflammatory protein. scleroma-like illness [5]. A relatively high proportion of individuals who ingested certain preparations of L-tryptophan developed a rare eosinophilia-myalgia syndrome $[6,7]$. The significance of these associations is entirely speculative but tryptophan is the only amino acid whose metabolism has been linked with these immunological disorders.

In humans, IDO expression has been observed by immunohistochemistry in placenta, tumor-draining nodes and primary tumors $[8,9]$. Both direct and indirect evidence indicate that IDO is widely expressed throughout the immune system, and more specifically, it is localized to a subset of cells with a macrophage or dendritic cell morphology $[10,11]$. These IDO-expressing cells are found at several sites of immune tolerance or privilege, including thymus, mucosa of the gut, epididymis, placenta, and the anterior chamber of the eyes [10, 12-14], and also has been shown to be expressed by human monocyte-derived macrophage [15] and dendritic cells (DCs) [16, 17]. In mice, IDO is important for maternal tolerance toward fetal alloantigens. Systemic administration of 1-methyl tryptophan (1MT), a pharmacological inhibitor of IDO, resulted in abortion of pregnant mice, demonstrating that IDO is a required 
component of the mechanisms by which the allogeneic fetus protects itself from rejection by the maternal immune system $[18,19]$. IDO can also mediate suppression of $\mathrm{T}$ cell immunity to MHC-mismatched liver allografts [20] and the control of $\mathrm{T}$ cell responses in autoimmune disorders [21]. Moreover, expression of IDO by immunogenic mouse tumor cells prevents their rejection by preimmunized mice $[8,22]$. Recent reports also suggest that IDO may also regulate the murine immunoregulatory CD8 dendritic cells [23]. These observations imply an essential role of IDO in regulating $\mathrm{T}$ cell-mediated immune responses, such as in the settings of transplantation and tumour tolerance [24].

Dendritic cells (DCs) are potent T cell stimulators, and act as sentinels against invasion of pathogens [25]. DCs can regulate the generation of Th1 and Th2 T cell immunity [26]. DCs are also important for the induction of peripheral tolerance [27]. Both immature and mature DCs constitutively express IDO protein, which suppresses T cell proliferation both in vitro and in vivo $[15,17]$. However, the functional activity of this enzyme may require additional signals. Recently it has been shown that ligation of the co-stimulatory molecules B7-1/B7-2 of DCs by human CD4 T cells modulates the IDO activity in DCs [28], indicating that $\mathrm{T}$ cells may function as a physiologic regulator for the expression of IDO in DCs. Other experiments have also demonstrated that inhibition of IDO activity with $1 \mathrm{MT}$ could lead to enhanced T cell proliferation in DC-T co-culture in vitro (for review, see ref [24]). Nevertheless, the effect of tryptophan deprivation on the modulation of DC function has not been determined. Our current study aimed to investigate the effect of tryptophan deprivation on the regulation of the phenotype, T cell stimulatory capacity, as well as chemotactic behaviour of DCs, which are essential for the induction of primary or T celldependent immunity.

\section{MATERIALS AND METHODS}

\section{Cells and DC activation}

Monocyte-derived cytokine-cultured dendritic cells were prepared as previously described [29]. Briefly, PBMCs were harvested from normal individuals and plated in 6-well plates for $2 \mathrm{~h}$. The nonadherent cells were subsequently washed away, and the remaining adherent cells were cultured in RPMI medium supplemented with $10 \%$ fetal calf serum, as well as $50 \mathrm{ng} / \mathrm{ml} \mathrm{GM-CSF}$ (Sandoz, Switzerland) and $50 \mathrm{ng} / \mathrm{ml}$ interleukin-4 (Peprotech, NJ, USA). RPMI also contained $50 \mu \mathrm{M}$ L-tryptophan. Half of the culture medium was replaced with fresh RPMI with GM-CSF and IL-4 on day-2, 4 and/or -6 before cell harvest. Day-6 or -7 DCs were further purified by two rounds of immunomagnetic depletion (Dynabeads, Dynal, Oslo, Norway) using monoclonal antibodies against CD3, CD8, CD14, CD16, CD19, and CD56 (all from BD PharMingen, San Diego, CA, USA), which yielded over $97 \%$ of cells expressing high levels of HLA-DR (data not shown). CD40 ligand transfected cells (CD40L Tf) were a kind gift from Dr Yong-Jun LIU, DNAX, Palo
Alto, USA. For DC activation, $1 \times 10^{5}$ purified DCs were exposed to $100 \mathrm{ng} / \mathrm{ml}$ LPS (Salmonella typhosa, Sigma, St Louis, USA) or 100 $\mathrm{ng} / \mathrm{ml}$ TNF- $\alpha$ (Peprotech, NJ, USA) for $3 \mathrm{~d}$, or co-cultured with CD40L Tf in 96 well plates at a ratio of 4 to 1 (DC vs CD40L transfectants) for $3 \mathrm{~d}$. Before co-culture, the CD40L Tf were treated with $50 \mu \mathrm{g} / \mathrm{ml}$ of mitomycin C (Sigma, St Louis, MO, USA) for 30 min at $37^{\circ} \mathrm{C}$, in order to prevent cellular proliferation. To study the effect of tryptophan deprivation, $2 \mathrm{mM}$ 1-methyl tryptophan (1MT, from Sigma, St Louis, USA) was added at one day before exposure to activation signals, or added into the culture at the same time with the exposure.

\section{Determination of intracellular expression of IDO}

DCs that had been treated with various combination of 1MT with activation stimuli were first treated with $10 \%$ normal goat serum, and then permeabilized and fixed by CytoPerm/CytoFix (Pharmingen, $\mathrm{BD}$, USA) as instructed by the manufacture. Cells were further stained with rabbit anti-IDO polyclonal antibody (Chemicon, CA, USA), followed by PE-conjugated goat anti-rabbit Ig, and then subjected to flow cytometry analysis (FACSCalibur instrument, Becton Dickinson, San Diego, CA, USA). Rabbit IgG was used as the corresponding isotype antibody control,

\section{Phenotypic study of DCs}

Phenotype of $1 \times 10^{5}$ purified DCs with or without treatment with 1MT was studied after activation with $100 \mathrm{ng} / \mathrm{ml}$ LPS (Salmonella typhosa, Sigma, St louis, USA), 100 ng/ml TNF- $\alpha$ (Peprotech, NJ, USA) or co-cultured with $2.5 \times 10^{4}$ CD40 ligand transfected L cells (CD40L L cells, a kind gift from Dr Yong-Jun LIU) by a FACSCalibur instrument (Becton Dickinson, San Diego, CA, USA) after staining with monoclonal antibodies against human CD80, CD83, CD86, CD40, HLA-DR, CCR5 and CXCR4, followed by FITC-conjugated secondary antibody (all from BD PharMingen, San Diego, CA, USA).

\section{T cell proliferative assay of DCs}

Responding T cell populations were prepared from PBMCs and treated with sodium periodate (BDH Chem. Co Ltd., Poole, UK) by incubating the cells at a final concentration of $10^{7} / \mathrm{ml}$ with sodium periodate $(0.25 \mathrm{mg} / \mathrm{ml}$ in PBS $)$ for $25 \mathrm{~min}$ at $4^{\circ} \mathrm{C}$. The cells were washed twice in complete media prior to use. Graded doses of irradiated $\left(5000 \mathrm{rad}^{137} \mathrm{Cs}\right) \mathrm{DCs}$ were added to allogeneic sodium periodatetreated T cells $\left(2 \times 10^{5} /\right.$ well $)$ in a final volume of $200 \mu \mathrm{l}$ in 96 well flatbottomed microtest wells. Proliferation in the wells was measured by adding $1 \mu \mathrm{Ci}$ of ${ }^{3} \mathrm{H}$-Thymidine/well after $22-26 \mathrm{~h}$. The cells were harvested 16-18 h later and counted on an LKB $\beta$ plate counter. Results were presented as mean \pm SD of triplicate cultures.

\section{Transwell chemotaxis assay}

$5-10 \times 10^{4}$ purified DCs were added to each transwell insert (Costar, Cambridge, MA, USA) containing $100 \mu$ culture medium above a polycarbonate membrane with $5 \mu \mathrm{m}$ pores. After $2 \mathrm{~h}$ incubation at $37^{\circ} \mathrm{C}$, the transwell inserts were lifted and the bases of the inserts were washed twice with $200 \mu 1$ ordinary medium to dislodge the cells that had just migrated through the multi-porous polycarbonate membrane but have not dropped into the lower chamber. The cells in the lower chamber then were collected, transferred into $5 \mathrm{ml} \mathrm{U}$-bottom clear tubes, and fixed with $5 \%$ formalin. The number of the migrated cells was determined by FACSCalibur instrument (Becton Dickinson, San Diego, CA, USA) as described [30]. Briefly, fluorescent beads (typically 50,000 beads) were added into the tubes as internal con- 
trol for cell counting. The acquisition of the cells was stopped after 5000 beads $(10 \%$ of the total) were acquired. The relative cell number of DCs in each tube then could be obtained by gating the large, granular cell population in the forward and side scatter dot plot. Results are expressed as total number of the migrated cells per transwell.

\section{Chemicals}

The reference compounds, L-tryptophan and L-kynurenine, were purchased from Sigma (St. Lois, MO, USA). Acetic acid, sodium acetate, potassium phosphates trichloroacetic acid and citric acid were obtained from Merck (Darmstadt, Germany). All chemicals used were of analytical grade. HPLC grade acetonitrile was obtained from Merck (Darmstadt, Germany).

\section{Sample Preparation}

Milimolar stock solutions of tryptophan and kynurenine were prepared by dissolving in $10 \%$ acetonitrile in potassium phosphate buffer $(0.05 \mathrm{M}, \mathrm{pH} 6.0)$ and stored at $-80^{\circ} \mathrm{C}$. These solutions were stable for at least six months. Working solutions of the external calibrator were prepared from freshly thawed stock solutions of tryptophan and kynurenine in various concentrations. $50 \mu 1$ of tryptophan, $10 \mu \mathrm{l}$ of kynurenine, and $940 \mu \mathrm{l}$ of albumin stock solution $(70 \mathrm{~g} / \mathrm{L}$ in double-distilled water) were mixed together. Aliquots of $200 \mu \mathrm{l}$ of calibrator preparation were then treated in the same way as the serum specimens.

Frozen medium specimens were thawed at room temperature. $200 \mu 1$ of medium were diluted with $200 \mu 1$ of potassium phosphate buffer $(0.05 \mathrm{mM}, \mathrm{pH} 6.0)$. Protein was precipitated with $50 \mu \mathrm{lof}$ trichloroacetic acid $(2 \mathrm{M})$. The capped tubes with the precipitate were immediately vortex-mixed and centrifuged for $10 \mathrm{~min}$ at 1,3000 $\mathrm{g}$ at $4^{\circ} \mathrm{C}$. Subsequently, $150 \mu \mathrm{l}$ of the supernatants were transferred into microvials (Chromacol) and placed into the autosampling device.

\section{Quantitative analysis of L-Trp and L-Kyn}

Quantitative analysis of tryptophan and kynurenine in the culture medium was performed using an automated Agilent 1100 Series HPLC system with quaternary pump, micro vacuum degasser, standard autosampler with a $100 \mu 1$ fixed sample loop, multiple wavelength (UV-Vis) detector and fluorescence detector. Tryptophan and its metabolites were separated with an analytical reversed phase $\mathrm{C} 18$ column (ZORBAX 300SB-C18, 250mm 4.6mm I.D.) with a reversed phase C18 guard cartridge. Aliquot $(50 \mu \mathrm{l})$ of supernatant was injected into column using programmable autosampler. Separation was achieved at $25^{\circ} \mathrm{C}$ by isocratic elution at a flow rate of $0.7 \mathrm{ml} / \mathrm{min}$. The mobile phase consisted of a $40 \mathrm{mM}$ sodium acetate/citric acid buffer solution in 5\% acetonitrile; the buffer solution was adjusted to $\mathrm{pH} 5$. 0 with $5 \mathrm{M} \mathrm{NaOH}$ prior to the addition of acetonitrile. Kynurenine was measured by UV detector at $360 \mathrm{~nm}$, while tryptophan was detected both by UV detector at $280 \mathrm{~nm}$ and fluorescence at an excitation wavelength of $286 \mathrm{~nm}$ and an emission wavelength of 366 $\mathrm{nm}$. Both detectors were connected in series to allow simultaneous measurements. Acquisition and integration of the chromatographic data were performed using a ChemStation base software. The concentration was determined as the integrated peak area measurement against external standards. Each sample was measured in triplicate; the averaged tryptophan and kynurenine concentrations and standard deviations were determined for all samples and compared with the concentrations calculated from the standards. Linear regressions were determined for both analyses, revealing a slope of 0.997 for tryptophan and 0.998 for kynurenine. The linear regression coefficients $\left(r^{2}\right)$ were $>0.998$.

\section{RESULTS}

Treatment with 1MT reduced intracellular expression of IDO in DCs after activation with LPS and CD40 ligation, and to a lesser extent to TNF- $\alpha$

To investigate the intracellular expression of IDO in DCs after tryptophan deprivation and cellular activation, DCs were treated with various stimuli for $3 \mathrm{~d}$ (group 1 cells), or pre-treated with $1 \mathrm{MT}$ one day before the $3 \mathrm{~d}$ stimulation (group 2 cells) or simultaneously with the exposure to stimuli (group 3 cells). Results revealed that immature DCs (iDC) constitutively expressed high levels of intracellular IDO (Fig. 1A, left), which was not affected by treatment with $1 \mathrm{MT}$ (Fig. 1A, right). Activation for $3 \mathrm{~d}$ with LPS, TNF- $\alpha$ or CD40 ligation up-regulated the expression of intracellular IDO in DCs prepared from in 3 of 5 donors (Fig. 1B, group 1 vs iDC in Fig. 1A). In the other 2 donors, the expression levels were relatively unchanged (data not shown). However, addition of 1MT one day before (group 2) or simultaneously with the exposure to LPS and CD40 ligation (group 3) significantly down-regulated the intracellular IDO levels (Fig. 1B and C, group 2 or 3 s group 1), and to a less extent, to TNF- $\alpha$ treatment.

\section{Treatment with 1MT inhibits the up-regulation of sur- face expression of co-stimulatory molecules on DCs induced by LPS and TNF- $\alpha$, and to a less extent, by CD40 ligation}

DCs exposed to LPS and TNF- $\alpha$ or co-cultured with CD40L Tf for $3 \mathrm{~d}$ significantly up-regulated the surface expression of CD80, CD86, HLA-DR, and the maturation marker CD83, and to a less extent, CD40 (Fig. 2A, B and $\mathrm{C}$, group 1 vs control). When DCs were treated with $1 \mathrm{MT}$ one day before exposure to LPS, the up-regulated expression of CD83, CD86, HLA-DR and CD80 was inhibited, at least in part, compared with cells treated with LPS alone (Fig. 2A, group 2 vs group 1). When 1MT was added into the cell culture at the same time as exposure to LPS, LPSinduced up-regulation of these molecules could also be partially inhibited, except that for CD40 (Fig. 2A, group 3 $v s$ group 1). Similarly, treatment with $1 \mathrm{MT}$ also resulted in inhibition of TNF- $\alpha$-induced up-regulation of CD83, CD86, HLA-DR and CD40 (Fig. 2B, group $2 v s$ group 1). In the cells activated by CD40 ligation (Fig. 2C), the expression of HLA-DR and CD83 was inhibited significantly by either prior or simultaneous treatment with 1MT (group 2 and 3 vs group 1, Fig. 2C). The expression of CD80 and CD86 induced by CD40 ligation was less inhibited by 1MT. 
A

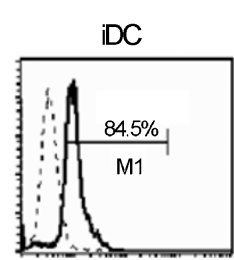

B

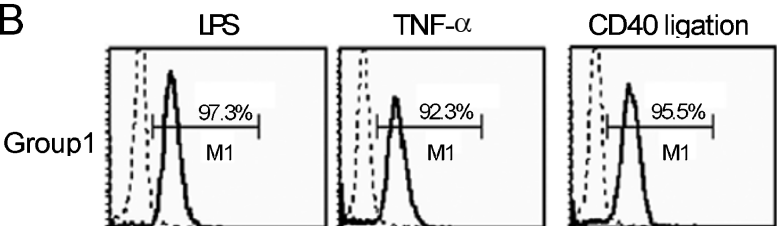

Group2
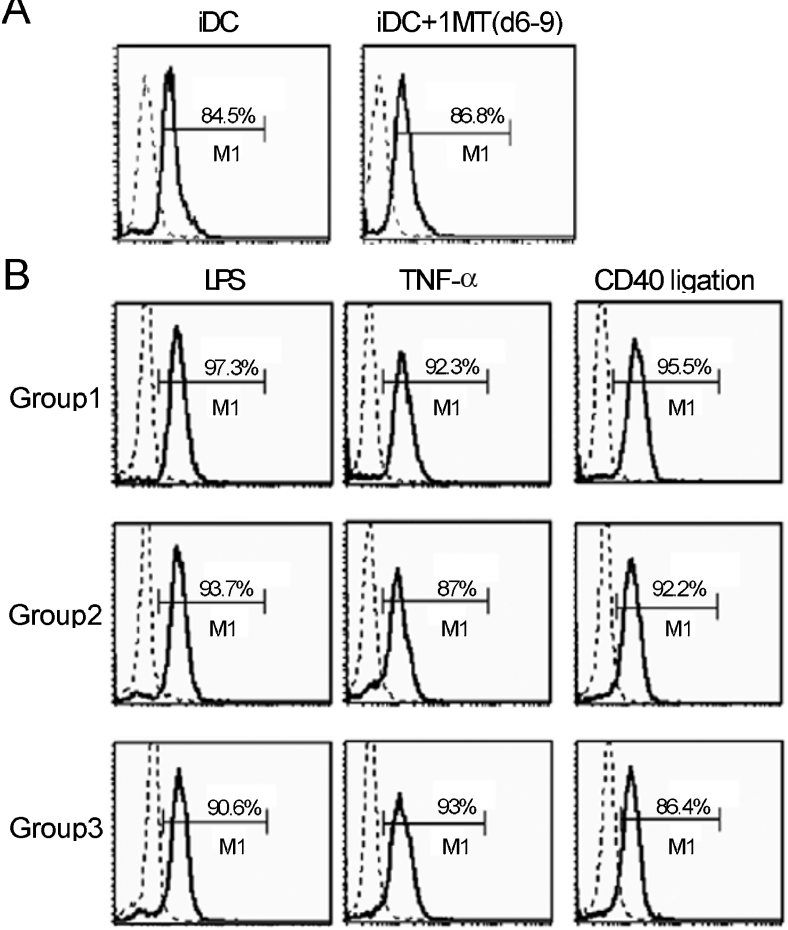

C

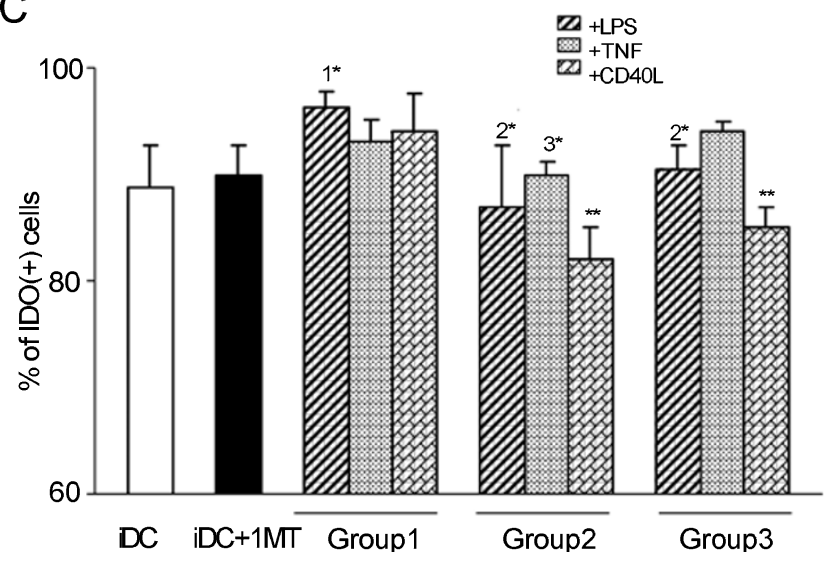

Fig. 1 Tryptophan deprivation reduced intracellular IDO expression of DCs after stimulation with LPS, TNF- $\alpha$ and CD40 ligation. (A) Intracellular IDO was constitutively expressed in iDC (left) and was unaffected by treatment with 1MT for $3 \mathrm{~d}$ (from $\mathrm{d} 6$ to $\mathrm{d} 9$ or $\mathrm{d}$ 7-d 10, right). Data are representative of 3 experiments. (B) Activation with maturational stimuli LPS, TNF- $\alpha$ and CD40L ligation for $3 \mathrm{~d}$ (d 6-9 or d 7-10) increased the IDO expression of DCs (group 1, $\mathrm{B} v \mathrm{~s}$, left). IDO expression was reduced significantly when cells were treated with $1 \mathrm{MT}$ on $\mathrm{d} 6$, followed by exposure to activation stimuli for $3 \mathrm{~d}$ (d 7-10; group 2 vs group 1). DCs treated simultaneously with $1 \mathrm{MT}$ and activation stimuli for $3 \mathrm{~d}(\mathrm{~d}$ 7-10) also had down-regulation of intracellular IDO (group $3 v s$ group 1), but to a less extant compared with group 2 cells. Data are representative of 3 experiments and are expressed as mean $\pm \mathrm{SD}$ in $(\mathbf{C}) .1 *: P<0.05$ compared with iDC; $2 *: P<0.05$ compared with LPS treatment in group $1 ; 3 * P<0.05$ compared with TNF- $\alpha$ treatment in group 1 ; $* * P<0.005$ compared with CD40L treatment in group 1 .
A
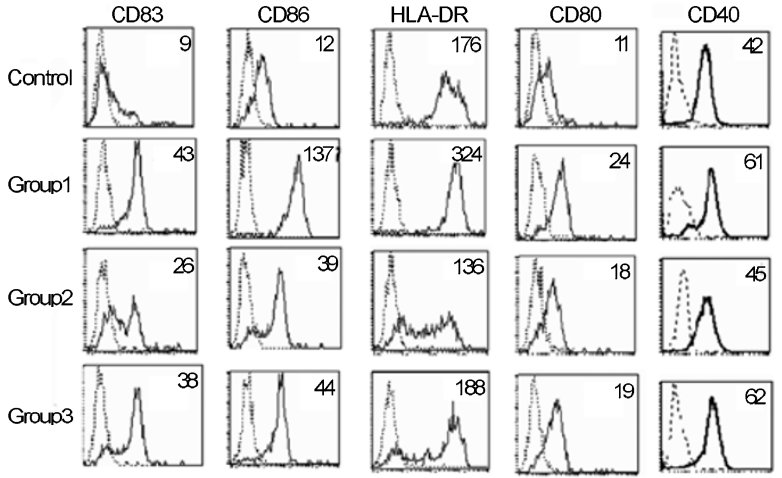

B
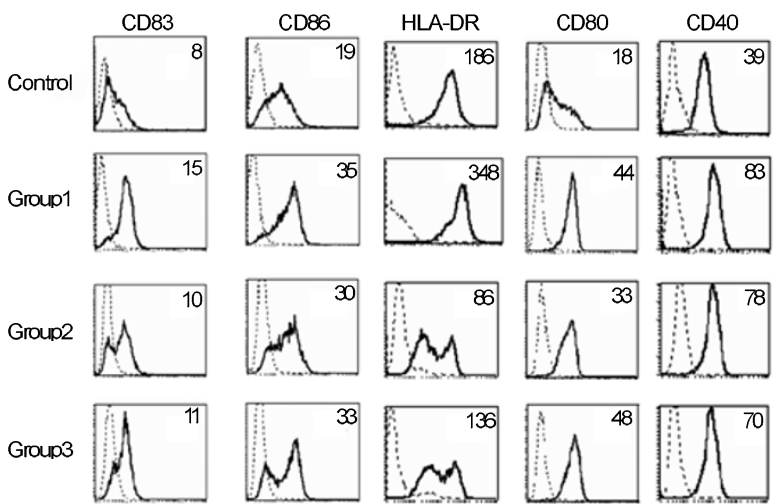

C
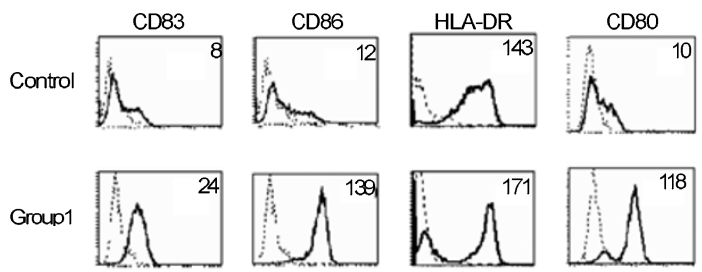

Group2
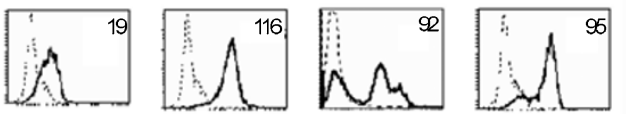

Group3
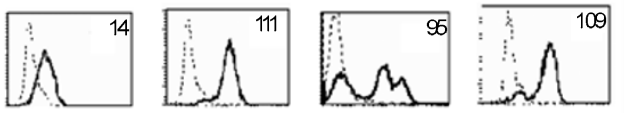

Fig. 2 1MT inhibited the up-regulation of the surface expression of CD86, CD83 and HLA-DR of human monocyte-derived DCs matured by LPS, TNF- $\alpha$ and CD40 ligation. Numbers at the right upper of the FACS profile indicates the mean fluorescence. (A) Phenotypic profile of CD83, CD86, HLA-DR, CD80 and CD40 of DCs after treatment with $100 \mathrm{ng} / \mathrm{ml} \mathrm{LPS} \mathrm{for} 3 \mathrm{~d}$ (group 1), or pre-treatment with $2 \mathrm{mM} 1 \mathrm{MT}$ one day before or simultaneous at the addition of LPS (defined as group 2 and 3 as in Fig. 1). CD83, CD86, HLA-DR and CD80 were significantly inhibited in group 2 and 3 compared with those in group 1. Down-regulation of CD40 expression by 1MT was less remarkable. (B) Phenotypic profile of DCs activated by $100 \mathrm{ng} / \mathrm{ml} \mathrm{TNF}-\alpha$ for $3 \mathrm{~d}$ without (group 1) or with treatment of 1-MT one day before or simultaneously (group 2 and 3 respectively) with the treatment with TNF- $\alpha$. (C) Phenotypic profile of DCs activated by co-culture with CD40L Tf for $3 \mathrm{~d}$ without (group 1) or with treatment of 1-MT 1 day before or simultaneously (group 2 and 3 respectively) with co-culture of CD40L Tf. 
Concentration of tryptophan was reduced while that of kyurenin was increased in culture supernatant of DCs after activation for $3 \mathrm{~d}$ in the presence of 1MT

In parallel to the experiments of Fig. 1 and 2, concentrations of tryptophan and its metabolite kyurenin was measured in the supernatant of DC culture at different time points. As shown in Fig. 3A, after activation with LPS alone for $3 \mathrm{~d}$, the tryptophan concentration was significantly reduced (Fig. 3A, group 1). By contrast, prior or simultaneous treatment with 1MT from d 6-9 significantly increased the concentration of tryptophan in the culture supernatant (group 2 and 3 cells vs group 1 and no treatment, Fig. 3A). Meanwhile, the concentration of kynurenin was reciprocally regulated, i.e., treatment with LPS alone increased the concentration of Kyn in the culture supernatant (group 1, Fig. 3A vs B). When 1MT was added before or simultaneously with exposure to LPS, the concentration of Kyn was not increased, indicating that Try was not metabolized through IDO pathway in the pres-
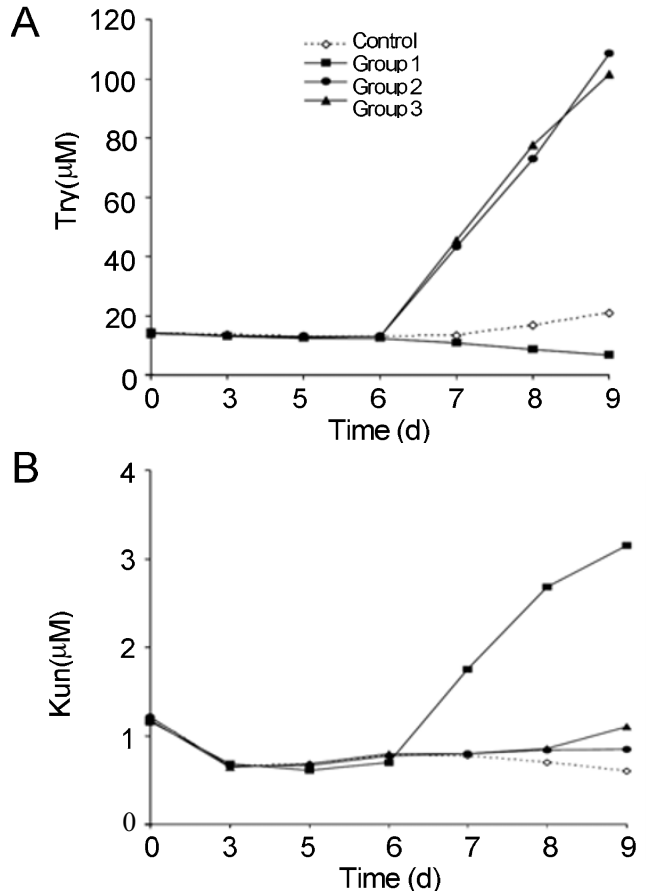

Fig. 3 Concentraion of tryptophan (Try) and kynurenin (Kyn) in the supernatant of DC culture treated by various combination of treatment with LPS and 1MT. (A) The concentration of Try in the culture supernatant remained constant until day 6 when cells were exposed to LPS for $3 \mathrm{~d}$ (group 1 cells). Addition of 1MT before or simultaneously with the exposure to LPS (group 2 and 3 respectively) prevented Try degradation and thus resulted in a high concentration of Try in the supernatant. (B) The concentration of Kyn was reciprocally regulated with that of Try. Data are representative of 3 independent experiments. Similar findings were made in DC exposure to TNF- $\alpha$ and CD40L Tf (data not shown). ence of 1MT. Results with activation by TNF- $\alpha$ and CD40 ligation gave similar findings (data not shown).

\section{Treatment with $1 \mathrm{MT}$ inhibited $\mathrm{T}$ cell stimulatory ca- pacity of DCs matured by LPS and TNF- $\alpha$, but not CD40 ligation}

In line with the phenotypic findings in Fig. 2, prior or simultaneous treatment of DCs with 1MT upon exposure to LPS and TNF- $\alpha$ significantly reduced T cell proliferation in 5-d DC-T co-culture (group 2 and $3 v s$ group 1, Fig. 4A and B), when compared with cells activated by

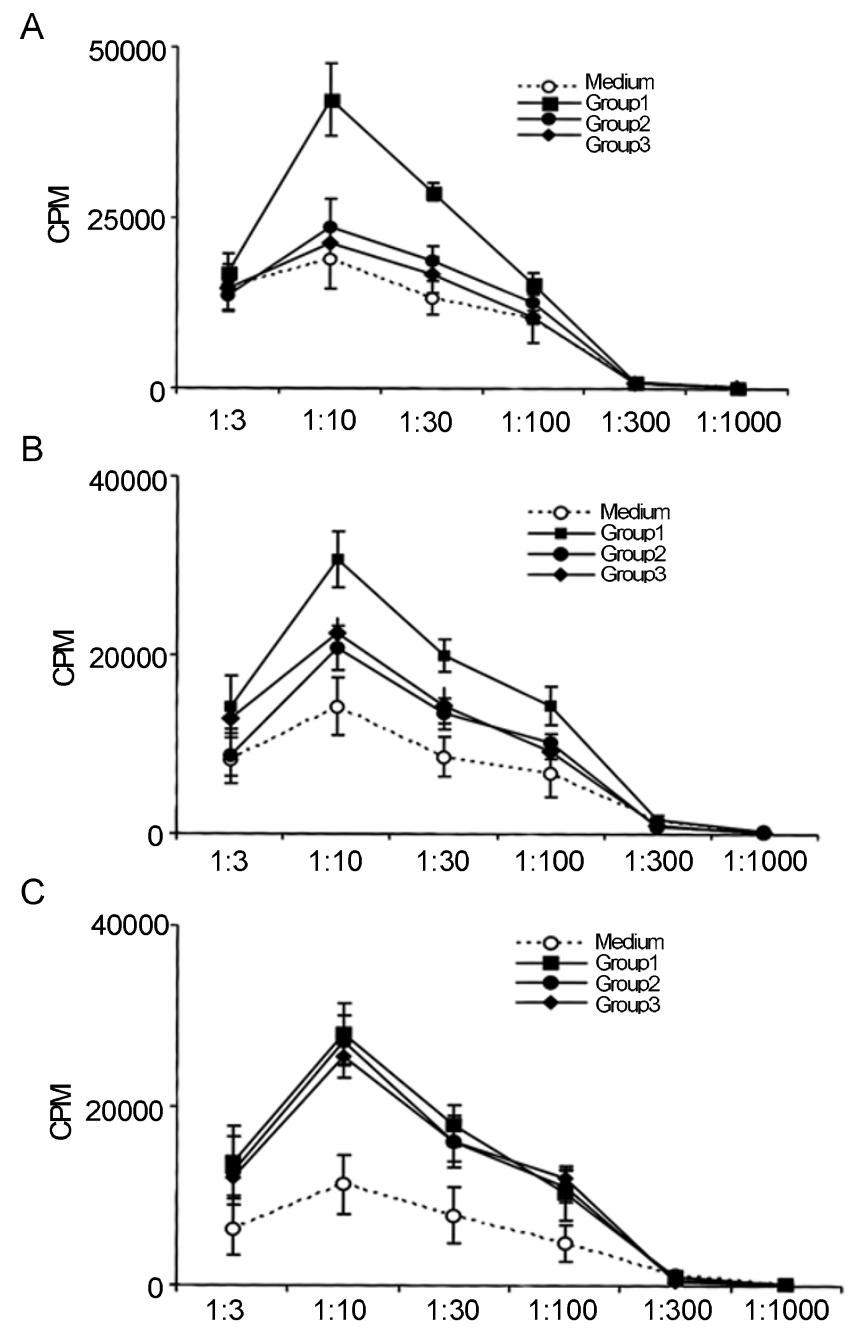

Fig. $4 \mathrm{~T}$ cell stimulatory capacity of DCs was reduced by the addition of 1MT before (group 2) or simultaneously (group 3) with the exposure to LPS (A) and TNF- $\alpha(\mathbf{B})$, but was relatively unaffected by CD40 ligation $(\mathbf{C})$. DCs activated by maturational stimuli alone (group 1) had a higher T cell stimulatory capacity, and addition of 1MT (group 2 and 3 ) significantly reduced the $\mathrm{T}$ cell proliferation in DC-T co-culture. Immature DCs cultured in ordinary medium was used as a negative control (labelled as "medium"). Data are expressed as mean $\pm \mathrm{SD}$ from 4 experiments. 
LPS or TNF- $\alpha$ alone (group 1). By contrast, the inhibition of $\mathrm{T}$ cell proliferative capacity was relatively unaf-

A
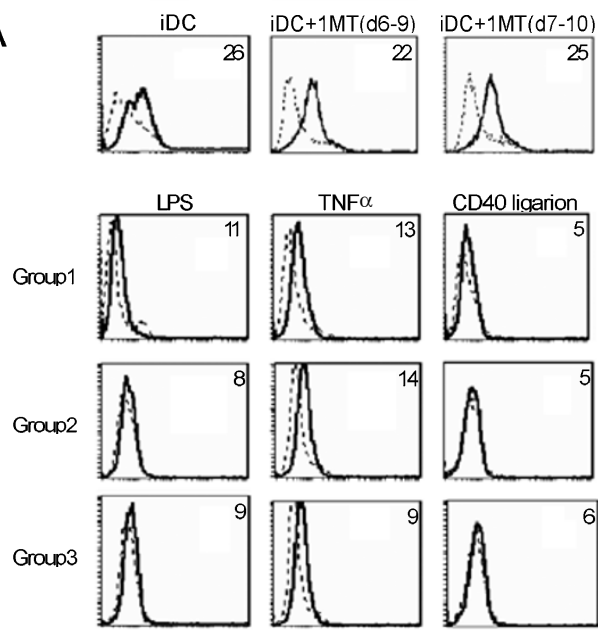

B
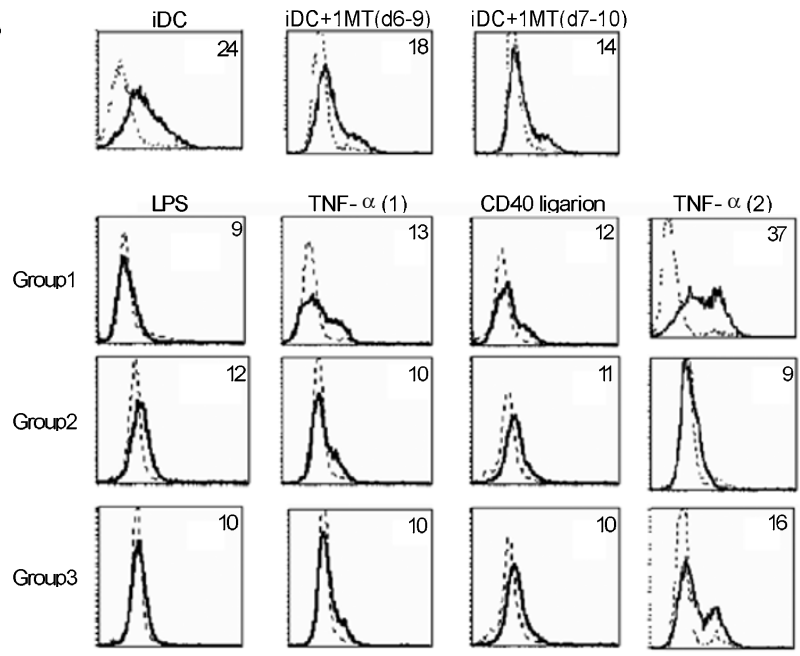

Fig. 5 Surface expression of chemokine receptor CCR5 and CXCR4 of DCs treated with various maturational stimuli with or without pre-treatment with 1MT. (A) Treatment with 1MT for $3 \mathrm{~d}$ (d 6-9 or d 7-10) did not affect the expression of CCR5 (upper panels). DC constitutively expressed CCR5 and CXCR4 ("iDC" in A and B). Exposure to LPS, TNF- $\alpha$ or CD40L transfectants, DCs significantly down-regulated the expression of CCR5 (group $1 v s$ iDC). Cells treated by 1MT before or simultaneously at the time of activation (group 2 and 3) also had a low expression of CCR5. Data are representative of 3 experiments. (B) Treatment with 1MT alone for $3 \mathrm{~d}$ down-regulated the CXCR4 expression (upper panels). Exposure to LPS, TNF- $\alpha$ and CD40L transfectants also resulted in down-regulation of CXCR4 (group $1 v s$ iDC), and addition of 1MT could not reverse this effect (group 2 and $3 ; n=3$ for LPS and CD40 ligation; $n=3 / 6$ for TNF- $\alpha$ (TNF- $\alpha(1))$. In $3 / 6$ experiments, TNF- $\alpha$ treatment up-regulated CXCR4 and addition of $1 \mathrm{MT}$ prevented such an effect (TNF- $\alpha$ (2) vs TNF- $\alpha$ (1)). fected by $1 \mathrm{MT}$ when DCs were activated by co-culture with CD40 ligand transfectants (Fig. 4C).

Treatment with 1MT down-regulated the surface expression of CXCR4 but not CCR5 of immature DCs

As shown in Fig. 5A and 5B, iDCs constitutively expressed CCR5 and CXCR4. Tryptophan deprivation by treatment with 1MT for $3 \mathrm{~d}$ (from d 6-9 or d 7-10) did not significantly affect the expression levels of CCR5 (Fig. $5 \mathrm{~A}$, upper panels). By contrast, CXCR4 was significantly reduced after exposure to $1 \mathrm{MT}$ for $3 \mathrm{~d}$ (Fig. 5B, upper panels). iDC activated by LPS, TNF- $\alpha$, or CD40 ligation for $3 \mathrm{~d}$ significantly down-regulated the CCR5 expression (Fig. 5A, group $1 v s$ iDC). Addition of 1MT, either prior to or simultaneous with activation by LPS, TNF- $\alpha$ or CD40 ligation, could not reverse the CCR5 expression (Fig. 5A, group 2 and 3 vs group 1). iDC treated with LPS or CD40 ligation also down-regulated the surface expression of CXCR4 (Fig. 5B, group 1 vs iDC). However, treatment of iDC with TNF- $\alpha$ resulted in two alternative outcomes: in 3 of 6 donors, TNF- $\alpha$ treatment down-regulated CXCR4 expression (Fig. 5B, TNF- $\alpha(1)$ ), whereas in the other 3 donors, CXCR 4 expression levels were up-regulated (Fig. $5 \mathrm{~B}, \mathrm{TNF}-\alpha(1)$ vs TNF- $\alpha(2))$. Nevertheless, the addition of $1 \mathrm{MT}$ consistently down-regulated the CXCR4 expression in all 6 donors after TNF- $\alpha$ activation (Fig. 5B, TNF (1) and $\mathrm{TNF}(2)$, group 2 and 3 vs group 1). It is also noted that in the 3 donors in which CXCR4 expression of DCs was up-regulated by TNF- $\alpha$ treatment, simultaneous treatment with 1MT in DC culture could at least in part reverse the CXCR4 surface levels (Fig. 5B, TNF- $\alpha(2)$, group 3).

\section{DC chemotaxis in response to chemokines correlated with the expression of chemokine receptors after 1MT treatment}

To investigate if $\mathrm{DC}$ responsiveness to chemokines was modulated by IDO, DC chemotaxis in response to various chemokines was studied in a transwell apparatus as described [30]. Results showed immature DCs migrated in response to CCR 5 ligands RANTES, MIP- $1 \alpha$ and MIP- $\beta$, as well as CXCR4 ligand SDF-1 $\alpha$. After activation with LPS, TNF- $\alpha$ and CD40 ligation, responsiveness to these chemokines was down-regulated (Fig. 6, group $1 v s$ medium, and SDF-1 $\alpha(1))$. Similar to the findings in CXCR 4 expression, in 3 of 6 donors TNF- $\alpha$ actually increased the responsiveness to SDF-1 $\alpha$ (Fig. 6, SDF- $1 \alpha$ (2), group $1 v s$ medium). Consistent with the findings in expression of CCR5 and CXCR4, treatment with 1MT, either prior to or simultaneous with exposure to activation stimuli, could not inhibit the down-regulation of the responsiveness to both CCR5 ligands and CXCR4 ligand. 


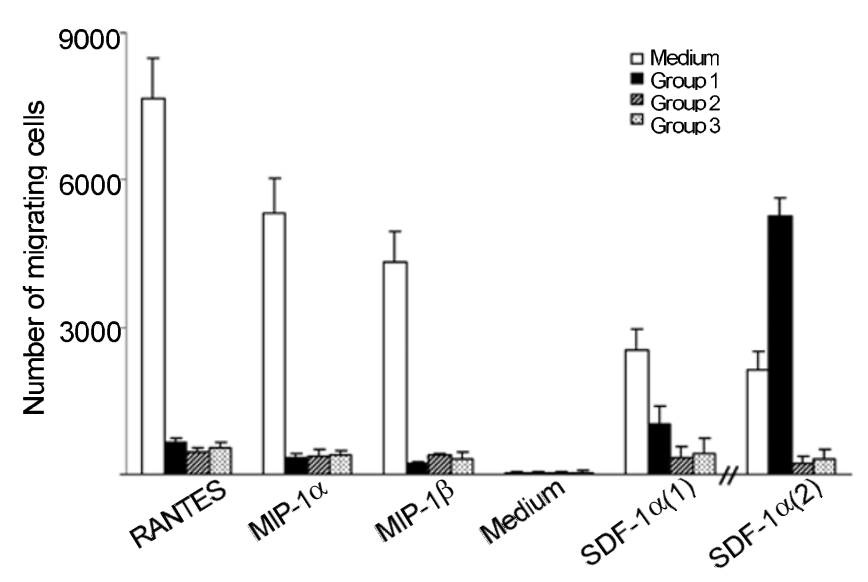

Fig. 6 Chemotaxis assay of DCs treated by TNF- $\alpha$ with or without addition of 1MT. Treatment with TNF- $\alpha$ for $3 \mathrm{~d}$ abolished the responsiveness to CCR5 ligands RANTES, MIP- $1 \alpha$, MIP- $\beta$, as well as to CXCR4 ligand SDF- $1 \alpha$ in $3 / 6$ experiments (group $1 v s$ medium, SDF-1 $\alpha(1))$. The addition of $1 \mathrm{MT}$ did not affect such an inhibition (group 2 and $3 v s$ group $1 ; n=3$ ). Activation by LPS and CD40L Tf with or without pre-treatment with 1MT also gave similar findings ( $n=3$, data not shown). In the other $3 / 6$ experiments, DCs treated with TNF- $\alpha$ had up-regulated responsiveness to SDF- $1 \alpha$, which could be prevented by the addition of $1 \mathrm{MT}$ (group 2 and $3 v s$ group 1, SDF-1 $\alpha(2))$.

\section{DISCUSSION}

Our data demonstrated that immature monocyte-derived DCs expressed high levels of IDO, as determined by intracellular staining. Furthermore, tryptophan deprivation by inclusion of 1-MT into DC culture did not alter the levels of IDO expression (Fig. 1A), suggesting that IDO expression is constitutively expressed in immature DCs and its levels were not affected by tryptophan deprivation. Furthermore, in selected donors DC activation by LPS, TNF- $\alpha$ or CD40 ligation actually up-regulated the intracellular IDO expression, although the percentage of IDOexpressing DCs before activation was already high ( $>80 \%$, Fig. 1A). In this regard, inconsistence was also observed by other groups that treatment of DCs with CD40 ligand or LPS did not consistently induce the expression of IDO mRNA [17]. However, in those selected donors in which IDO was up-regulated after activation, addition of 1MT prior to or simultaneously with exposure to activation stimuli could inhibit the up-regulated IDO expression, with a more remarkable effect upon stimulation by LPS and CD40 ligation (group 2 and $3 v s$ group 1, Fig. 1B and C) and a less inhibitory effect upon treatment with TNF- $\alpha$. The reason for such a discrepance in DC preparations from different donors is unclear but it seems to suggest that there might be a negative feedback for the regulation of IDO expression in DCs after encountering various activa- tion stimuli.

It was also demonstrated in our study that the up-regulated expression of co-stimulatory molecules such as CD86, CD80 and MHC class II after DC activation, as well as the DC maturation marker CD83, were also significantly inhibited when 1MT was added before or simultaneously with exposure to LPS (group 2 and $3 v s$ group 1, Fig. 2A). Similarly, the up-regulated expression of CD83, CD86, and HLA-DR was also inhibited by 1MT when exposed to TNF- $\alpha$, and the up-regulated expression of HLA-DR was also inhibited when stimulated with TNF- $\alpha$ and CD40 ligation (Fig. $2 \mathrm{~B}$ and C). The inhibition of CD40L-induced up-regulation of CD83, CD86, CD80 was, however, less remarkable in comparison to activation by LPS and TNF- $\alpha$. Furthermore, activation with LPS indeed reduced the levels of tryptophan in the culture supernatant (Fig. 3A, group 1 vs control), and prior or simultaneous addition of 1MT could inhibit the use of tryptophan, manifested as a higher concentration of tryptophan in the culture media on day 9 (Fig. 3A, group 2 and 3 vs group 1). By contrast, the levels of Kynurenin was reciprocally regulated (Fig. 3B), indicating that tryptophan metabolism was indeed inhibited in the presence of IDO inhibitor in these experiments. Taken together, these results revealed that in the absence of tryptophan metabolism through kynurenin pathway, i.e., tryptophan deprivation, phenotypic maturation of DCs activated by these stimuli was partially inhibited, suggesting that DC activation was dependent on tryptophan utilization. It has been reported that IDO production by DCs resulted in the inhibition of $\mathrm{T}$ cell proliferation through the catabolism of tryptophan [17], and that kynurenin pathway metabolites are also active in suppressing $\mathrm{T}$ cell proliferation, with apoptosis being the proposed mechanism [31]. However, no excessive apoptosis of DCs were observed in our study after DCs activation with different stimuli. Therefore, it is likely that while IDO-mediated suppression of T cell proliferation may result from tryptophan deprivation in the culture system and/or from the toxicity of the kynurenin pathway metabolites, 1MT-mediated inhibition of the phenotypic activation of DCs by various stimuli may simply reflect the absence of sufficient amount of tryptophan for DC activation.

To determine if the inhibition of the up-regulated phenotypic markers for DC activation correlates with the function, $\mathrm{T}$ cell proliferation assays were examined in coculture of T cells with DCs stimulated with maturation stimuli in the presence or absence of 1MT. Results showed that DCs activated by LPS, TNF- $\alpha$ and CD40 ligation exhibited a more potent $\mathrm{T}$ cells stimulatory capacity, compared with immature DCs (Fig. 4A, B and C, group 1 vs medium group). However, treatment with $1 \mathrm{MT}$ before or 
simultaneously with the exposure to LPS and TNF- $\alpha$ significantly indeed reduced their $\mathrm{T}$ cell stimulatory capacity when compared with cells activated in the absence of 1MT (Fig. 4A and B, group 2 and 3 vs group 1). These results are consistent with the phenotypic study in which the upregulated expression of certain important co-stimulatory molecules of activated DCs was also inhibited by $1 \mathrm{MT}$ (Fig. 2A and B). Interestingly, treatment of CD40L-activated DCs with $1 \mathrm{MT}$ before or simultaneous with the stimulation did not result in reduction of $\mathrm{T}$ cell stimulatory capacity (Fig. 4C, group 2 and $3 v s$ group 1). This observation is consistent with the regulation of phenotypic markers where, except HLA-DR, expression of CD83, CD86 and CD40 of DCs induced by CD40 ligation was less inhibited by 1MT, compared with cells treated by LPS or TNF- $\alpha$ (Fig. 2C vs A and B). These results suggest that DC activation by pathogenic stimuli or inflammatory cytokine (e.g., LPS from Gram(-) bacteria and TNF- $\alpha$ ) may be more dependent on the tryptophan than by the physiological stimuli CD40 ligand (e.g., from activated CD4 $\mathrm{T}$ cells). It is therefore likely that tryptophan deprivation is more important for DC-mediated defense mechanisms against pathogens/inflammation, but to a lesser extent in a more physiological condition, e.g., when DCs migrate to lymph nodes and encounter activated CD4 T cells for the induction of $\mathrm{T}$ cell immunity. These findings seems to contradict previous observations that addition of $1 \mathrm{MT}$ in the DC-T co-culture in vitro enhanced $\mathrm{T}$ cell proliferation $[3,5]$. The reason for this remains to be determined; however, it is likely that in previous reports, 1MT that had been directly added into the DC-T co-culture would also inhibit the IDO effect on both DCs and T cells, whereas in our system only DCs were treated with IDO inhibitor and the tryptophan utilization of T cells was not interfered. It has been noticed that low tryptophan concentrations are associated with inhibited proliferation of viruses [32], protozoa parasites $[2,33]$ and other pathogens [34] in eukaryotic cells. Our findings may therefore suggest that tryptophan deprivation not only inhibits activities of pathogens but also reduces $\mathrm{T}$ cell stimulatory capacity of DCs after exposure to LPS or TNF- $\alpha$ that may be abundant in the site of infection or inflammation. These results may indicate that the intensity of DC-dependent antigen-specific immunity is tightly regulated by the local concentrations of tryptophan, i.e., T cell stimulatory capacity of DCs may be reduced when levels of antigens in the microenvironment are low, which may contribute to a delicate balance between the $\mathrm{T}$ cell immunity and the local antigenic concentrations.

The capacity of DC to initiate immune responses is highly dependent on their specialized migratory and tissue homing properties. We have previously shown that imma- ture DCs exhibit potent chemotaxis to the $\mathrm{CC}$ chemokines macrophage inflammatory protein (MIP)- $1 \alpha$, MIP- $1 \beta$, RANTES, and a weak response to the CXC chemokine stromal cell-derived factor (SDF)-1 $\alpha$ [30]. We have also shown that maturation of DCs from most donors induced by TNF- $\alpha$ reduced or abolished responsiveness to the former $\mathrm{CC}$ chemokines but enhanced responsiveness to SDF-1 $\alpha$ in selected individuals (Fig. 6). Furthermore, this phenomenon correlates with changes in chemokine receptor expression: CCR5 expression was reduced while CXCR4 expression was enhanced (Fig. 5B, group 1 of TNF- $\alpha(2) v s$ control). In this study, our data also demonstrated that treatment with 1MT either prior to or simultaneous with LPS activation or CD40 ligation could not reverse the down-regulated expression of CCR5 and CXCR4 (Fig. 5A and B, group 2 and 3 vs group 1). In selected individuals ( 3 out of 6 donors), treatment with TNF- $\alpha$ induced up-regulation of the CXCR4 (TNF- $\alpha(2)$, Fig. 5B), which was virtually completely prevented by treatment with $1 \mathrm{MT}$ one day prior to exposure to TNF- $\alpha$, and partially but significantly when 1MT was added at the same time with TNF- $\alpha$. Overall, the effect of 1MT was inhibitory on the expression of CCR5 and CXCR4 that are important co-receptors for HIV-1 infection (CCR5 for M-tropic HIV1, and CXCR4 for T-tropic) of DCs [29]. Whether or not these findings may also contribute to the susceptibility of HIV infection of DCs remains to be investigated.

The functional study also confirmed that the alteration of the surface expression of chemokine receptors on DCs after 1MT treatment correlated with their migratory characteristics in response to chemokines. The reduction in the responsiveness to SDF-1 $\alpha$ after 1MT treatment was also demonstrated in all donors, no matter these cells had an increased or reduced responsiveness to SDF- $1 \alpha$ after activation by TNF- $\alpha$ (Fig. 6 , SDF- $1 \alpha(1)$ and SDF- $1 \alpha$ (2), group $1 v s$ iDC). These results indicate that tryptophan deprivation by $1 \mathrm{MT}$ also had a inhibitory effect in the expression of chemokine receptors and chemotactic responsiveness of DCs to chemokines, apart from the regulation for the expression of co-stimulatory molecules (Fig. 2) and the T cell stimulatory capacity (Fig. 4).

In summary, our data suggest that while tryptophan deprivation in DC-T co-culture could enhance T cell proliferation, as demonstrated by addition of $1 \mathrm{MT}$ to inhibit IDO function [35], the effect of tryptophan deprivation on DC per se in vitro was actually inhibitory during activation by either pathogenic stimuli (e.g., LPS or TNF$\alpha$ ) or physiological stumulus (e.g., CD40 ligation). We therefore conclude that DC activation and chemotaxis behavior is, at least in part, tryptophan-dependent, which may subsequently contribute to the initiation and regulation of $\mathrm{DC}$-mediated $\mathrm{T}$ cell responses. 
Received, July 2, 2004

Revised, Jan 18, 2005

Accepted, Jan 25,2005

\section{REFERENCES}

1 Taylor MW, Feng GS. Relationship between interferon-gamma, indoleamine 2,3-dioxygenase, and tryptophan catabolism. Faseb J 1991; 5:2516-22.

2 Pfefferkorn ER. Interferon gamma blocks the growth of Toxoplasma gondii in human fibroblasts by inducing the host cells to degrade tryptophan. Proc Natl Acad Sci USA 1984; 81:908-12.

3 Gupta SL, Carlin JM, Pyati P, et al. Antiparasitic and antiproliferative effects of indoleamine 2,3-dioxygenase enzyme expression in human fibroblasts. Infect Immun 1994; 2:2277-84.

4 Aune TM, Pogue SL. Inhibition of tumor cell growth by interferon-gamma is mediated by two distinct mechanisms dependent upon oxygen tension: induction of tryptophan degradation and depletion of intracellular nicotinamide adenine dinucleotide. J Clin Invest 1989; 84:863-75.

5 Sternberg EM, Van Woert MH, Young SN, et al. Development of a scleroderma-like illness during therapy with L-5-hydroxytryptophan and carbidopa. N Engl J Med 1980; 303:782-7.

6 Belongia EA, Hedberg CW, Gleich GJ, et al. An investigation of the cause of the eosinophilia-myalgia syndrome associated with tryptophan use. N Engl J Med 1990; 323:357-65.

7 Mayeno AN, Lin F, Foote CS, et al. Characterization of "peak E" a novel amino acid associated with eosinophilia-myalgia syndrome. Science 1990; 250:1707-8.

8 Uyttenhove C, Pilotte L, Theate I, et al. Evidence for a tumoral immune resistance mechanism based on tryptophan degradation by indoleamine 2,3-dioxygenase. Nat Med 2003; 9:1269-74.

9 Lee JR, Dalton RR, Messina JL, et al. Pattern of recruitment of immunoregulatory antigen-presenting cells in malignant melanoma. Lab Invest 2003; 83:1457-66.

10 Moffett JR, Espey MG, Namboodiri MA. Antibodies to quinolinic acid and the determination of its cellular distribution within the rat immune system. Cell Tissue Res 1994; 278:461-9.

11 Espey MG, Tang Y, Morse HC 3rd, Moffett JR, Namboodiri MA. Localization of quinolinic acid in the murine AIDS model of retrovirus-induced immunodeficiency: implications for neurotoxicity and dendritic cell immunopathogenesis. Aids 1996; 10: 151-8.

12 Yoshida R, Nukiwa T, Watanabe Y, et al. Regulation of indoleamine 2,3-dioxygenase activity in the small intestine and the epididymis of mice. Arch Biochem Biophys 1980; 203:343-51.

13 Yoshida R, Urade Y, Nakata K, Watanabe Y, Hayaishi O. Specific induction of indoleamine 2,3-dioxygenase by bacterial lipopolysaccharide in the mouse lung. Arch Biochem Biophys 1981; 212:629-37.

14 Malina HZ, Martin XD. Indoleamine 2,3-dioxygenase: antioxidant enzyme in the human eye. Graefes Arch Clin Exp Ophthalmol 1996; 234:457-62.

15 Munn D.H, Shafizadeh E, Attwood JT, et al. Inhibition of T cell proliferation by macrophage tryptophan catabolism. J Exp Med 1999; 189:1363-72.

16 Munn DH, Sharma MD, Lee JR, et al. Potential regulatory function of human dendritic cells expressing indoleamine 2,3dioxygenase. Science 2002; 297:1867-70.
17 Hwu P, Du MX, Lapointe R, et al. Indoleamine 2,3-dioxygenase production by human dendritic cells results in the inhibition of $\mathrm{T}$ cell proliferation. J Immunol 2000; 164:3596-9.

18 Munn DH, Zhou M, Attwood JT, et al. Prevention of allogeneic fetal rejection by tryptophan catabolism. Science 1998; 281: 1191-3.

19 Mellor AL, Sivakumar J, Chandler P, et al. Prevention of T celldriven complement activation and inflammation by tryptophan catabolism during pregnancy. Nat Immunol 2001; 2:64-8.

20 Miki T, Sun H, Lee Y, et al. Blockade of tryptophan catabolism prevents spontaneous tolerogenicity of liver allografts. Transplant Proc 2001; 33:129-30.

21 Sakurai K, Zou JP, Tschetter JR, Ward JM, Shearer GM. Effect of indoleamine 2,3-dioxygenase on induction of experimental autoimmune encephalomyelitis. J Neuroimmunol 2002; 129:18696.

22 Friberg M, Jennings R, Alsarraj M, et al. Indoleamine 2,3dioxygenase contributes to tumor cell evasion of $\mathrm{T}$ cell-mediated rejection. Int J Cancer 2002; 101:151-5.

23 Grohmann U, Fallarino F, Bianchi R, et al. IL-6 inhibits the tolerogenic function of $\mathrm{CD} 8$ alpha + dendritic cells expressing indoleamine 2,3-dioxygenase. J Immunol 2001; 167:708-14.

24 Munn DH, Mellor AL. IDO and tolerance to tumors. Trends Mol Med 2004; 10:15-8.

25 Chung NP, Chen Y, Chan VS, Tam PK, Lin CL. Dendritic cells: sentinels against pathogens. Histol Histopathol 2004; 19:317-24.

26 Rissoan MC, Soumelis V, Kadowaki N, et al. Reciprocal control of T helper cell and dendritic cell differentiation. Science 1999; 283:1183-6.

27 Banchereau J, Steinman RM. Dendritic cells and the control of immunity. Nature 1998; 392:245-52.

28 Munn DH, Sharma MD, Mellor AL. Ligation of B7-1/B7-2 by human $\mathrm{CD} 4(+) \mathrm{T}$ cells triggers indoleamine 2,3-dioxygenase activity in dendritic cells. J Immunol 2004; 172:4100-10.

29 Lin CL, Sewell AK, Gao GF, et al. Macrophage-tropic HIV induces and exploits dendritic cell chemotaxis. J Exp Med 2000; 192:587-94.

30 Lin CL, Suri RM, Rahdon RA, Austyn JM, Roake JA. Dendritic cell chemotaxis and transendothelial migration are induced by distinct chemokines and are regulated on maturation. Eur $\mathbf{J}$ Immunol 1998; 28:4114-22.

31 Fallarino F, Grohmann U, Vacca C, et al. T cell apoptosis by tryptophan catabolism. Cell Death Differ 2002; 9:1069-77.

32 Bodaghi B, Goureau O, Zipeto D, et al. Role of IFN-gammainduced indoleamine 2,3 dioxygenase and inducible nitric oxide synthase in the replication of human cytomegalovirus in retinal pigment epithelial cells. J Immunol 1999; 162:957-64.

33 Nagineni CN, Pardhasaradhi K, Martins MC, Detrick B, Hooks JJ. Mechanisms of interferon-induced inhibition of Toxoplasma gondii replication in human retinal pigment epithelial cells. Infect Immun 1996; 64:4188-96.

34 Byrne GI, Lehmann LK, Landry GJ. Induction of tryptophan catabolism is the mechanism for gamma-interferon-mediated inhibition of intracellular Chlamydia psittaci replication in T24 cells. Infect Immun 1986; 53:347-51.

35 Terness P, Bauer TM, Rose L, et al. Inhibition of allogeneic T cell proliferation by indoleamine 2,3-dioxygenase-expressing dendritic cells: mediation of suppression by tryptophan metabolites. J Exp Med 2002; 196:447-57. 